\title{
Eosinophilic fasciitis associated with L-tryptophan ingestion
}

\author{
Maurice E Hamilton
}

\begin{abstract}
A 62 year old woman taking L-tryptophan developed eosinophilic fasciitis shortly after starting an exercise class. She received prednisone without benefit but improved after azathioprine treatment was started and Ltryptophan was discontinued. As products containing L-tryptophan have recently been implicated in development of the eosinophiliamyalgia syndrome it is suggested that the use of L-tryptophan might have contributed to the development of eosinophilic fasciitis in this patient. Similarities with toxic oil syndrome are noted. Additional studies are warranted to determine the prevalence of L-tryptophan ingestion among patients diagnosed as having eosinophilic fasciitis.
\end{abstract}

Eosinophilia-myalgia syndrome, an illness characterised by the presence of eosinophilia $\left(\geqslant 10^{9}\right.$ cells $\left./ 1\right)$ and generalised myalgias not explained by infection or neoplasm, has recently been linked to the ingestion of products containing L-tryptophan. ${ }^{1}$ Other reported manifestations of this syndrome include ascending polyneuropathy and vasculitis. I wish to report the development of eosinophilic fasciitis in a patient taking L-tryptophan.

University of California, Davis, School of Medicine

M E Hamilton

Correspondence to: Dr Maurice E Hamilton, 534 East Pine Street, Stockton, CA 95204, USA.

Accepted for publication 28 June 1990

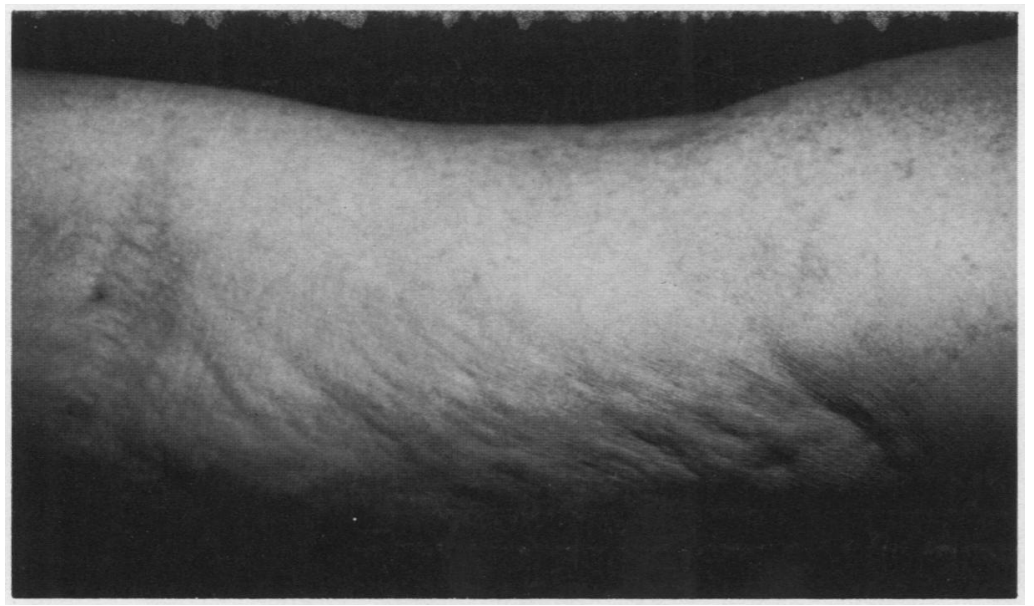

Induration of proximal right arm; note 'puckered' appearance, typical of eosinophilic fasciitis.
Raynaud's phenomenon or dysphagia. The patient had started an exercise programme at a fitness centre two weeks before the onset of her illness. Past history showed that she had hypertension treated with captopril. Since 1987 she had taken L-tryptophan (Energen, Norwalk, CA) 2000-3000 mg daily for relaxation.

Examination was remarkable for swelling and tenderness of the hands, forearms, thighs, and legs; the subcutaneous tissue of these areas was indurated and the skin appeared dimpled (peau d'orange). Mild pitting oedema of the legs was present. Tenderness was noted over the metacarpophalangeal and proximal interphalangeal joints, wrists, shoulders, and ankles. Subcutaneous nodules, sclerodactyly, digital pitting, telangiectasia, and contractures were absent.

Clinical suspicion of eosinophilic fasciitis led to the performance of a complete blood count, which showed white blood cell count $10.9 \times 10^{9}$ cells $/ 1$ with $35 \%$ eosinophils. Erythrocyte sedimentation rate was $18 \mathrm{~mm} / \mathrm{h}$. A biopsy specimen (from skin to muscle) of the left thigh showed striking oedema and fibrosis of septa in the subcutaneous fat and of the fascia. The dermis displayed a dense, predominantly lymphocytic, infiltrate. A similar infiltrate was present in the subcutaneous fat and fascia. These changes were consistent with eosinophilic fasciitis.

The patient was treated with prednisone (initially $40 \mathrm{mg}$ daily and later $70 \mathrm{mg}$ daily) without benefit. The induration progressed to affect the proximal right arm, which appeared 'puckered' (figure). Multiple papules, which evolved into ulcers, developed on the left leg; a biopsy showed dermal sclerosis with focal ulceration but no vasculitis. Treatment with azathioprine (100 mg daily) was started and prednisone was tapered and discontinued; the patient stopped using L-tryptophan. The myalgias resolved, the ulcers healed, and the induration of the arms and legs improved. The azathioprine dose is now being tapered.

\section{Discussion}

Eosinophilic fasciitis is a scleroderma-like disease characterised by tender swelling and induration of the subcutaneous tissue of the arms and legs. A full thickness biopsy of affected tissue shows sclerosis of deep fascia, subcutis, and dermis with prominent lymphocytic infiltration; plasma cells, eosinophils, and histiocytes may also be present. ${ }^{2}$ Laboratory studies usually show eosinophilia and hypergammaglobulinaemia. Calcinosis, Raynaud's phenomenon, digital pitting, telangiectasia, and visceral 
involvement-features typical of sclerodermaare usually absent, as in our patient. ${ }^{3}$ The development of eosinophilic fasciitis in our patient within two weeks of beginning an exercise programme is consistent with previous observations that physical exertion may precipitate the onset of this syndrome. Most patients with eosinophilic fasciitis improve with moderate doses of prednisone, in contrast with this patient.

The recently described association between the ingestion of products containing L-tryptophan and the development of eosinophilia with myalgias suggests that the use of L-tryptophan might have been a significant factor in the development of eosinophilic fasciitis in our patient. Whether the continued use of L-tryptophan made our patient's disease more refractory to treatment with the usual doses of corticosteroids is speculative. Also uncertain is the degree to which starting treatment with azathioprine $v$ discontinuing L-tryptophan contributed to her clinical improvement.

The mechanism whereby L-tryptophan, an essential amino acid, or its metabolites may induce eosinophilia-myalgia syndrome and eosinophilic fasciitis is unknown. The illness that developed in our patient shares certain features with the toxic oil syndrome that occurred after ingestion of adulterated rapeseed oil in Spain. ${ }^{4}$ During the early phase of this syndrome patients presented with tender swelling and induration of the arms and legs associated with peripheral blood eosinophilia. Histological studies showed mononuclear cell infiltrates and eosinophils in the dermis, fascia, muscle, and vessel walls. Recently, Varga and coworkers also described the development of diffuse fasciitis with eosinophilia during L-tryptophan treatment; they showed increased expression of type I procollagen gene in dermal and fascial fibroblasts from two such patients. ${ }^{5}$

The recent popularity of products containing $\mathbf{L}$-tryptophan may explain, in part, the increasing recognition of eosinophilic fasciitis. Additional studies are warranted to determine the prevalence of L-tryptophan ingestion among patients diagnosed as having eosinophilic fasciitis. 1 Eosinophilia-myalgia syndrome and L-tryptophan-containing York, 1989. MMWR 1989; 38: 785-8.

2 Moutsopoulos H M, Webber B L, Pavlidis N A, Fostiropoulos G, Goules D, Shulman L E. Diffuse fasciitis with eosinophilia: a clinicopathologic study. Am $\mathcal{J}$ Med 1980; 68: 701-9.

3 Lakhanpal S, Ginsburg W W, Michet C J, Doyle I A, Moore S B. Eosinophilic fasciitis: clinical spectrum and therapeutic $S$ B. Eosinophilic fasciitis: clinical spectrum and therapeutic
response in 52 cases. Semin Arthritis Rheum 1988; 17: response

4 Alonso-Ruiz A, Zea-Mendoza A C, Salazar-Vallinas J M, Rocamora-Ripoll A, Beltran-Gutierrez J. Toxic oil syndrome: a syndrome with features overlapping those of various forms of scleroderma. Semin Arthritis Rheum 1986; 15: 200-12.

5 Varga J, Peltonen J, Uitto J, Jimenez S. Development of diffuse fasciitis with eosinophilia during L-tryptophan treatment: demonstration of elevated type I collagen gene expression in affected tissues. Ann Intern Med 1990; 112 344-51. 